\title{
Quantitative unique continuation for the heat equations with inverse square potential
}

\author{
Guojie Zheng ${ }^{1 *}$, Keqiang $\mathrm{Li}^{1}$ and Yuanyuan Zhang ${ }^{2}$
}

"Correspondence:

guojiezheng@whu.edu.cn

${ }^{1}$ College of Mathematics and Information Science, Henan Normal

University, Xinxiang, P.R. China Full list of author information is

available at the end of the article

\section{空 Springer}

\begin{abstract}
In this paper, we investigate the unique continuation properties for multi-dimensional heat equations with inverse square potential in a bounded convex domain $\Omega$ of $\mathbb{R}^{d}$. We establish observation estimates for solutions of equations. Our result shows that the value of the solutions can be determined uniquely by their value on an open subset $\omega$ of $\Omega$ at any given positive time $L$.
\end{abstract}

MSC: $35 \mathrm{~K} 67$

Keywords: Heat equations; Singular potential; Unique continuation; Frequency function

\section{Introduction}

In this paper, we consider the quantitative unique continuation for multi-dimensional heat equations with a singular potential term. The heat equations studied in this article are described by

$$
\begin{cases}\partial_{t} \varphi(x, t)-\Delta \varphi(x, t)-V(x) \varphi(x, t)=0 & \text { in } \Omega \times(0, L], \\ \varphi(x, t)=0 & \text { on } \partial \Omega \times(0, L], \\ \varphi(x, 0)=\varphi_{0}(x) & \text { in } \Omega,\end{cases}
$$

where $L$ is a positive number, $\Omega \subset \mathbb{R}^{d}(d \geq 3)$ is a convex and bounded domain with smooth boundary $\partial \Omega$ and $x=0 \in \Omega$. The potential function is

$$
V(x)=\frac{\mu}{|x|^{2}}, \quad \mu<\mu_{*}=\frac{(d-2)^{2}}{4} .
$$

The well-posedness theory of these equations have mainly been studied in recent years. For the existence and other properties of solutions to equation (1.1), we refer to [2, 3, 7 , $13,19]$. In particular, in [3], authors proved that if a non-negative initial value $\varphi_{0} \in L^{2}(\Omega)$ is prescribed, then there exists a unique global weak solution for equation (1.1) under assumption (1.2), but as $\mu>\mu_{*}$, the local solution may not exist. In [19], the wellposedness of equation (1.1) without the sign restriction for the solution is thoroughly discussed. In summary, for any initial value $\varphi_{0} \in L^{2}(\Omega)$, there exists a unique solution $\varphi \in C\left([0, T] ; L^{2}(\Omega)\right) \cap L^{2}\left(0, T ; H_{0}^{1}(\Omega)\right)$ for equation (1.1) with (1.2). Throughout the paper,

(c) The Author(s) 2018. This article is distributed under the terms of the Creative Commons Attribution 4.0 International License (http://creativecommons.org/licenses/by/4.0/), which permits unrestricted use, distribution, and reproduction in any medium, provided you give appropriate credit to the original author(s) and the source, provide a link to the Creative Commons license, and indicate if changes were made. 
we use $\|\cdot\|$ and $\langle\cdot, \cdot\rangle$ to denote the usual norm and the inner product in the space $L^{2}(\Omega)$, respectively. Besides, variables $x$ and $t$ for functions of $(x, t)$ and variable $x$ for functions of $x$ will be omitted, provided that it is not going to cause any confusion.

The main results are presented as follows.

Theorem 1.1 Suppose that $\omega$ is a non-empty open subset of $\Omega, 0 \in \omega$, and $\varphi_{0} \in L^{2}(\Omega)$. Then there exist two positive numbers $\alpha=\alpha(\Omega, \omega), C=C(\Omega, \omega)$ such that, for each $L>0$,

$$
\int_{\Omega}|\varphi(x, L)|^{2} d x \leq C e^{\frac{C}{L}}\left(\int_{\Omega}\left|\varphi_{0}\right|^{2} d x\right)^{1-\alpha}\left(\int_{\omega}|\varphi(x, L)|^{2} d x\right)^{\alpha}
$$

Moreover, if $\varphi_{0} \neq 0$, then

$$
\left\|\varphi_{0}\right\|_{H^{-1}(\Omega)}^{2} \leq C \exp \left(\frac{C}{L}+C L \frac{\|\varphi(x, 0)\|_{L^{2}(\Omega)}^{2}}{\|\varphi(x, 0)\|_{H^{-1}(\Omega)}^{2}}\right) \int_{\omega}|\varphi(L)|^{2} d x
$$

\section{Remark 1.1}

(i) The mathematical model (1.1) is a special case where potential term $V(x)=\lambda /|x|^{2}$. The singular potentials occur in many physical phenomena. In non-relativistic quantum mechanics, the harmonic oscillator and the Coulomb central potential are typical examples of such kind (see [12]). In particular, it can also be found in the study of quantum scattering theory (see [17]). Thus, it is very significant to study the properties of equation (1.1).

(ii) The constant $C$ in (1.3) or (1.4) stands for a positive constant only depending on $\Omega$ and $\omega$. Specifically, it depends on the size of $\omega$ and $\Omega$, and the distance from $\omega$ to $\partial \Omega$.

(iii) These results demonstrate that solutions of (1.1) can be uniquely determined by its value on an open subset $\omega$, which contains zero, at any given positive time $L$.

The study of unique continuation for the solutions of PDEs began at the beginning of the last century. It plays an important role in PDEs theory, inverse problems, and control theory. To the best of our knowledge, the first result for strong unique continuation of parabolic equations was derived in 1974 in [10]. In [10], the authors established the unique continuation for parabolic equations with time independent coefficients by the properties of eigenfunctions of the corresponding elliptic operator, and this approach cannot be applied to parabolic equations with time dependent coefficients. From 1980s, there have been more results of unique continuation for parabolic equations, and we refer the readers to $[5,8,9,11,14-16]$ and rich references cited therein. In our paper, we mainly study this property for the heat equations with the inverse square potential. The main difficulty in proving Theorem 1.1 lies in the singular potential terms. This difficulty is overcome by setting up a new norm for $H_{0}^{1}(\Omega)$ in terms of the Hardy-Poincaré inequality. With the aid of the frequency function, we can obtain those quantitative estimates.

We organize this paper as follows: In Sect. 2, we give some preliminary results; Sect. 3 is devoted to the proof of Theorem 1.1.

\section{Preliminary results}

We suppose that $\Omega \subset \mathbb{R}^{d}(d \geq 3)$ is an open domain with a smooth boundary $\partial \Omega$ and $0 \in \Omega$. Let us first recall the well-known Hardy-Poincaré inequality that there exists a 
positive constant $C(\Omega)$, which only depends on $\Omega$, such that

$$
\int_{\Omega}\left[|\nabla v(x)|^{2}-\mu_{*} \frac{v^{2}(x)}{|x|^{2}}\right] d x \geq C(\Omega) \int_{\Omega} v^{2}(x) d x, \quad \forall v \in H_{0}^{1}(\Omega)
$$

where $\mu_{*}$ is provided in (1.2). The proof for inequality (2.1) can be found in [4, 13]. Furthermore, as $\mu<\mu_{*}$,

$$
\int_{\Omega}\left[|\nabla v(x)|^{2}-\mu \frac{v^{2}(x)}{|x|^{2}}\right] d x \geq C(\Omega)\left(1-\frac{\mu}{\mu_{*}}\right) \int_{\Omega}|\nabla v(x)|^{2} d x+\frac{C(\Omega) \mu}{\mu_{*}} \int_{\Omega} v^{2}(x) d x .
$$

By (2.2), we can equip $H_{0}^{1}(\Omega)$ with the following inner product:

$$
\langle f, g\rangle_{H_{0}^{1}(\Omega)}=\int_{\Omega}[\nabla f(x) \cdot \nabla g(x)-V(x) f(x) g(x)] d x, \quad \forall f, g \in H_{0}^{1}(\Omega)
$$

and the norm $\|f\|_{H_{0}^{1}(\Omega)}=\left(\int_{\Omega}\left(|\nabla f|^{2}-V(x) v^{2}\right) d x\right)^{\frac{1}{2}}$ is equivalent to the standard norm in $H_{0}^{1}(\Omega)$. Taking $L^{2}(\Omega)$ as a pivot space, we have the following compact embeddings (see [18]):

$$
H_{0}^{1}(\Omega) \hookrightarrow L^{2}(\Omega) \hookrightarrow H^{-1}(\Omega)
$$

and

$$
\langle f, g\rangle_{H^{-1}(\Omega), H_{0}^{1}(\Omega)}=\langle f, g\rangle_{L^{2}(\Omega)}, \quad \forall f \in L^{2}(\Omega), g \in H_{0}^{1}(\Omega)
$$

For each $\lambda>0$, we define the following weight function over $\mathbb{R}^{d} \times[0, L]$ :

$$
G_{\lambda}(x, t)=\frac{1}{(L-t+\lambda)^{d / 2}} e^{-\frac{|x|^{2}}{4(L-t+\lambda)}}
$$

Then, for each $t \in[0, L]$, we define the following three functions over the interval $[0, L]$ :

$$
\begin{aligned}
& H_{\lambda}(t)=\int_{\Omega}|\varphi(x, t)|^{2} G_{\lambda}(x, t) d x, \\
& D_{\lambda}(t)=\int_{\Omega}\left[|\nabla \varphi(x, t)|^{2}-\frac{\mu|\varphi(x, t)|^{2}}{|x|^{2}}\right] G_{\lambda}(x, t) d x,
\end{aligned}
$$

and

$$
N_{\lambda}(t)=\frac{2 D_{\lambda}(t)}{H_{\lambda}(t)}
$$

where $\varphi(x, t)$ is the solution of equation (1.1). The function $N_{\lambda}(t)$ was first discussed in [1]. It was called frequency function (see also [5, 6], and [16]). In this article, we define a different frequency function based on the new norm of $H_{0}^{1}(\Omega)$. We always suppose $H_{\lambda}(t) \neq 0$. Now, we will discuss the properties for the functions $G_{\lambda}(x, t)$. 
Lemma 2.1 For each $\lambda>0$, the function $G_{\lambda}$ given in (2.5) has the following identities over $\mathbb{R}^{d} \times[0, L]:$

$$
\begin{aligned}
& \partial_{t} G_{\lambda}(x, t)+\triangle G_{\lambda}(x, t)=0, \\
& \nabla G_{\lambda}(x, t)=\frac{-x}{2(L-t+\lambda)} G_{\lambda}(x, t), \\
& \partial_{i}^{2} G_{\lambda}(x, t)=\frac{-1}{2(L-t+\lambda)} G_{\lambda}(x, t)+\frac{\left|x_{i}\right|^{2}}{4(L-t+\lambda)^{2}} G_{\lambda}(x, t),
\end{aligned}
$$

and for $i \neq j$,

$$
\partial_{i} \partial_{j} G_{\lambda}(x, t)=\frac{x_{i} x_{j}}{4(L-t+\lambda)^{2}} G_{\lambda}(x, t) .
$$

Next, we will study the properties for derivatives of the functions $H_{\lambda}(t), D_{\lambda}(t)$, and $N_{\lambda}(t)$ in the following lemmas.

Lemma 2.2 For any $\lambda>0$, the following identity holds:

$$
H_{\lambda}^{\prime}(t)=-2 D_{\lambda}(t)
$$

and

$$
H_{\lambda}^{\prime}(t)=2 \int_{\Omega} \varphi\left(\partial_{t} \varphi-\nabla \varphi \frac{x}{2(L-t+\lambda)}\right) G_{\lambda} d x .
$$

Proof By direct computation, we obtain

$$
\begin{aligned}
H_{\lambda}^{\prime}(t) & =2 \int_{\Omega} \varphi \partial_{t} \varphi G_{\lambda} d x+\int_{\Omega}|\varphi|^{2} \partial_{t} G_{\lambda} d x \\
& =2 \int_{\Omega} \varphi \partial_{t} \varphi G_{\lambda} d x-\int_{\Omega}|\varphi|^{2} \triangle G_{\lambda} d x \\
& =2 \int_{\Omega} \varphi\left(\partial_{t} \varphi-\Delta \varphi\right) G_{\lambda} d x-2 \int_{\Omega}|\nabla \varphi|^{2} G_{\lambda} d x \\
& =-2 \int_{\Omega}\left(\nabla|\varphi|^{2}-\frac{\mu \varphi^{2}}{|x|^{2}}\right) G_{\lambda} d x=-2 D_{\lambda}(t) .
\end{aligned}
$$

Second,

$$
\begin{aligned}
H_{\lambda}^{\prime}(t) & =2 \int_{\Omega} \varphi \partial_{t} \varphi G_{\lambda} d x-\int_{\Omega}|\varphi|^{2} \triangle G_{\lambda} d x \\
& =2 \int_{\Omega} \varphi \partial_{t} \varphi G_{\lambda} d x+\int_{\Omega} \nabla|\varphi|^{2} \nabla G_{\lambda} d x \\
& =2 \int_{\Omega} \varphi\left(\partial_{t} \varphi-\nabla \varphi \frac{x}{2(L-t+\lambda)}\right) G_{\lambda} d x .
\end{aligned}
$$

This completes the proof of this lemma. 
Remark 2.1 By Lemma 2.2, we have

$$
D_{\lambda}(t)=-\int_{\Omega} \varphi\left(\partial_{t} \varphi-\nabla \varphi \frac{x}{2(L-t+\lambda)}\right) G_{\lambda} d x .
$$

Lemma 2.3 For any $\lambda>0$, the following identity holds:

$$
D_{\lambda}^{\prime}(t)=-\theta-2 \int_{\Omega}\left(\partial_{t} \varphi-\frac{x}{2(L-t+\lambda)} \nabla \varphi\right)^{2} G_{\lambda} d x+\frac{1}{L-t+\lambda} D_{\lambda}(t)
$$

where

$$
\theta=\int_{\partial \Omega}|\nabla \varphi|^{2} \frac{\partial G_{\lambda}}{\partial \nu} d \sigma-2 \int_{\partial \Omega} \frac{\partial \varphi}{\partial \nu}\left(\nabla \varphi \nabla G_{\lambda}\right) d \sigma \geq 0 .
$$

Here and in what follows, $v$ is the outward unit normal vector of the surface $\partial \Omega$.

Proof By the fact $\varphi=0$ on $\partial \Omega$, we first derive that

$$
\begin{aligned}
D_{\lambda}^{\prime}(t)= & 2 \int_{\Omega} \nabla \varphi \nabla \partial_{t} \varphi G_{\lambda} d x-\int_{\Omega} \frac{2 \mu \varphi \partial_{t} \varphi}{|x|^{2}} G_{\lambda} d x+\int_{\Omega}\left[|\nabla \varphi|^{2}-\frac{\mu \varphi^{2}}{|x|^{2}}\right] \partial_{t} G_{\lambda} d x \\
= & 2 \int_{\Omega} \operatorname{div}\left(\partial_{t} \varphi \nabla \varphi G_{\lambda}\right) d x-2 \int_{\Omega} \partial_{t} \varphi \operatorname{div}\left(\nabla \varphi G_{\lambda}\right) d x \\
& -2 \int_{\Omega} \frac{\mu \varphi \partial_{t} \varphi}{|x|^{2}}-\int_{\Omega}\left[|\nabla \varphi|^{2}-\frac{\mu \varphi^{2}}{|x|^{2}}\right] \triangle G_{\lambda} d x \\
= & -2 \int_{\Omega} \partial_{t} \varphi \Delta \varphi G_{\lambda} d x-2 \int_{\Omega} \partial_{t} \varphi \nabla \varphi \nabla G_{\lambda} d x \\
& -2 \int_{\Omega} \partial_{t} \varphi \frac{\mu \varphi}{|x|^{2}} G_{\lambda} d x-\int_{\Omega}\left[\left.\nabla \varphi\right|^{2}-\frac{\mu \varphi^{2}}{|x|^{2}}\right] \triangle G_{\lambda} d x \\
= & -2 \int_{\Omega} \partial_{t} \varphi\left(\Delta \varphi+\frac{\mu \varphi}{|x|^{2}}\right)_{\lambda} d x-2 \int_{\Omega} \partial_{t} \varphi \nabla \varphi \frac{-x}{2(L-t+\lambda)} G_{\lambda} d x \\
& -\int_{\Omega}\left[|\nabla \varphi|^{2}-\frac{\mu \varphi^{2}}{|x|^{2}}\right] \triangle G_{\lambda} d x \\
= & -2 \int_{\Omega}\left(\partial_{t} \varphi\right)^{2} G_{\lambda} d x-2 \int_{\Omega} \partial_{t} \varphi \nabla \varphi \frac{-x}{2(L-t+\lambda)} G_{\lambda} d x \\
& -\int_{\Omega}\left[\left.\nabla \varphi\right|^{2}-\frac{\mu \varphi^{2}}{|x|^{2}}\right] \triangle G_{\lambda} d x .
\end{aligned}
$$

Now, we deal with the last term in (2.18). In fact,

$$
\begin{aligned}
\int_{\Omega}|\nabla \varphi|^{2} \triangle G_{\lambda} d x= & \int_{\partial \Omega}|\nabla \varphi|^{2} \frac{\partial G_{\lambda}}{\partial v} d \sigma-\int_{\Omega} \nabla|\nabla \varphi|^{2} \nabla G_{\lambda} d x \\
= & \int_{\partial \Omega}|\nabla \varphi|^{2} \frac{\partial G_{\lambda}}{\partial v} d \sigma-2 \int_{\Omega} \nabla \varphi \nabla\left(\nabla \varphi \nabla G_{\lambda}\right) d x \\
& +2 \sum_{i=1}^{d} \int_{\Omega} \partial_{i} \varphi\left(\nabla \varphi \partial_{i} \nabla G_{\lambda}\right) d x \\
= & \int_{\partial \Omega}|\nabla \varphi|^{2} \frac{\partial G_{\lambda}}{\partial v} d \sigma-2 \int_{\Omega} \operatorname{div}\left[\nabla \varphi\left(\nabla \varphi \nabla G_{\lambda}\right)\right] d x
\end{aligned}
$$




$$
\begin{aligned}
& \quad+2 \int_{\Omega} \Delta \varphi\left(\nabla \varphi \nabla G_{\lambda}\right) d x+2 \sum_{i=1}^{d} \int_{\Omega} \partial_{i} \varphi\left(\nabla \varphi \partial_{i} \nabla G_{\lambda}\right) \\
& =\int_{\partial \Omega}|\nabla \varphi|^{2} \frac{\partial G_{\lambda}}{\partial \nu} d \sigma-2 \int_{\partial \Omega} \frac{\partial \varphi}{\partial \nu}\left(\nabla \varphi \nabla G_{\lambda}\right) d \sigma \\
& +2 \int_{\Omega} \triangle \varphi\left(\nabla \varphi \nabla G_{\lambda}\right) d x+2 \sum_{i=1}^{d} \int_{\Omega} \partial_{i} \varphi\left(\nabla \varphi \partial_{i} \nabla G_{\lambda}\right) .
\end{aligned}
$$

Thus,

$$
\begin{aligned}
\int_{\Omega}|\nabla \varphi|^{2} \triangle G_{\lambda} d x= & \theta+2 \int_{\Omega} \Delta \varphi\left(\nabla \varphi \nabla G_{\lambda}\right) d x+2 \sum_{i=1}^{d} \int_{\Omega} \partial_{i} \varphi\left(\nabla \varphi \partial_{i} \nabla G_{\lambda}\right) \\
= & \theta+2 \int_{\Omega} \Delta \varphi\left(\nabla \varphi \nabla G_{\lambda}\right) d x-\int_{\Omega}|\nabla \varphi|^{2} \frac{1}{L-t+\lambda} G_{\lambda} d x \\
& +2 \int_{\Omega}\left(\frac{x}{2(L-t+\lambda)} \nabla \varphi\right)^{2} G_{\lambda} d x
\end{aligned}
$$

where

$$
\theta=\int_{\partial \Omega}|\nabla \varphi|^{2} \frac{\partial G_{\lambda}}{\partial \nu} d \sigma-2 \int_{\partial \Omega} \frac{\partial \varphi}{\partial \nu}\left(\nabla \varphi \nabla G_{\lambda}\right) d \sigma .
$$

Meanwhile,

$$
\begin{aligned}
\int_{\Omega} \frac{\mu \varphi^{2}}{|x|^{2}} \Delta G_{\lambda} d x & =-\int_{\Omega} \nabla \frac{\mu \varphi^{2}}{|x|^{2}} \nabla G_{\lambda} d x \\
& =-\int_{\Omega} \frac{2 \mu \varphi \nabla \varphi}{|x|^{2}} \nabla G_{\lambda} d x+\int_{\Omega} \frac{2 \mu \varphi^{2} x}{|x|^{4}} \nabla G_{\lambda} d x \\
& =-\int_{\Omega} \frac{2 \mu \varphi \nabla \varphi}{|x|^{2}} \nabla G_{\lambda} d x-\frac{1}{L-t+\lambda} \int_{\Omega} \frac{\mu \varphi^{2}}{|x|^{2}} G_{\lambda} d x
\end{aligned}
$$

Combining it with (2.18), (2.19), (2.21) indicates

$$
\begin{aligned}
D_{\lambda}^{\prime}(t)= & -2 \int_{\Omega}\left(\partial_{t} \varphi\right)^{2} G_{\lambda} d x-2 \int_{\Omega} \partial_{t} \varphi \nabla \varphi \frac{-x}{2(L-t+\lambda)} G_{\lambda} d x \\
& -\theta-2 \int_{\Omega} \Delta \varphi\left(\nabla \varphi \nabla G_{\lambda}\right) d x-2 \int_{\Omega}\left(\frac{x}{2(L-t+\lambda)} \nabla \varphi\right)^{2} G_{\lambda} d x \\
& +\int_{\Omega}|\nabla \varphi|^{2} \frac{1}{L-t+\lambda} G_{\lambda} d x \\
& -\int_{\Omega} \frac{2 \mu \varphi \nabla \varphi}{|x|^{2}} \nabla G_{\lambda} d x-\frac{1}{L-t+\lambda} \int_{\Omega} \frac{\mu \varphi^{2}}{|x|^{2}} G_{\lambda} d x \\
= & -2 \int_{\Omega}\left(\partial_{t} \varphi\right)^{2} G_{\lambda} d x-4 \int_{\Omega} \partial_{t} \varphi \nabla \varphi \frac{-x}{2(L-t+\lambda)} G_{\lambda} d x \\
& -2 \int_{\Omega}\left(\frac{x}{2(L-t+\lambda)} \nabla \varphi\right)^{2} G_{\lambda} d x-\theta+\frac{1}{L-t+\lambda} D_{\lambda}(t) \\
= & -\theta-2 \int_{\Omega}\left(\partial_{t} \varphi-\frac{x}{2(L-t+\lambda)} \nabla \varphi\right)^{2} G_{\lambda} d x+\frac{1}{L-t+\lambda} D_{\lambda}(t) .
\end{aligned}
$$


Next, we will prove $\theta \geq 0$. Since $\varphi=0$ on $\partial \varphi$, it holds that $\nabla \varphi=\frac{\partial \varphi}{\partial \nu} \nu$. For the domain $\Omega$ is convex and $0 \in \Omega$, we have $x \cdot v \geq 0$. This, together with (2.7) and (2.20), shows that

$$
\begin{aligned}
\theta & =-\frac{1}{2(L-t+\lambda)} \int_{\partial \Omega}|\nabla \varphi|^{2}(x \cdot v) G_{\lambda} d \sigma+\frac{1}{L-t+\lambda} \int_{\partial \Omega}\left|\frac{\partial \varphi}{\partial \nu}\right|^{2}(x \cdot v) G_{\lambda} d \sigma \\
& =\frac{1}{2(L-t+\lambda)} \int_{\partial \Omega}|\nabla \varphi|^{2}(x \cdot v) G_{\lambda} d \sigma \geq 0 .
\end{aligned}
$$

This completes the proof of this lemma.

The frequency function $N_{\lambda}(t)$ satisfies the following lemma.

Lemma 2.4 For any $\lambda>0$,

$$
\lambda N_{\lambda}(L) \leq(L-t+\lambda) N_{\lambda}(t) \leq(L+\lambda) N_{\lambda}(0), \quad t \in[0, L] .
$$

Proof By Lemmas 2.2, 2.3, and Remark 2.1, we derive

$$
\begin{aligned}
N_{\lambda}^{\prime}(t)= & \frac{2}{H_{\lambda}^{2}(t)}\left\{D_{\lambda}^{\prime}(t) H_{\lambda}(t)-H_{\lambda}^{\prime}(t) D_{\lambda}(t)\right\} \\
= & \frac{2}{H_{\lambda}^{2}(t)}\left\{\left[-\theta-2 \int_{\Omega}\left(\partial_{t} \varphi-\frac{x}{2(L-t+\lambda)} \nabla \varphi\right)^{2} G_{\lambda} d x+\frac{1}{L-t+\lambda} D_{\lambda}(t)\right]\right. \\
& \left.\times \int_{\Omega} \varphi^{2} G_{\lambda} d x+2\left(\int_{\Omega} \varphi\left(\partial_{t} \varphi-\nabla \varphi \frac{x}{2(L-t+\lambda)}\right) G_{\lambda} d x\right)^{2}\right\} \\
\leq & \frac{1}{L-t+\lambda} N_{\lambda} .
\end{aligned}
$$

The last step is based on the Cauchy-Schwarz inequality. It shows that

$$
\left[(L-t+\lambda) N_{\lambda}(t)\right]^{\prime} \leq 0
$$

Thus, $(L-t+\lambda) N_{\lambda}(t)$ is a decreasing function, and

$$
\lambda N_{\lambda}(L) \leq(L-t+\lambda) N_{\lambda}(t) \leq(L+\lambda) N_{\lambda}(0), \quad t \in[0, L] .
$$

This completes the proof of this lemma.

Letting $m=\sup _{x \in \Omega}\|x\|_{\mathbb{R}^{d}}^{2}$, we have the following.

Lemma 2.5 For any $\lambda>0$,

$$
\lambda N_{\lambda}(L) \leq\left(1+\frac{\lambda}{L}\right)\left[\frac{m}{L}+2 \ln \frac{\int_{\Omega}|\varphi(x, 0)|^{2} d x}{\int_{\Omega}|\varphi(x, L)|^{2} d x}\right]
$$

Proof We first have

$$
\frac{L}{2} \lambda N_{\lambda}(L)=\int_{0}^{\frac{L}{2}} \lambda N_{\lambda}(L) d t
$$


It follows from Lemma 2.4 that

$$
\frac{L}{2} \lambda N_{\lambda}(L) \leq(L+\lambda) \int_{0}^{\frac{L}{2}} N_{\lambda}(t) d t=(L+\lambda) \int_{0}^{\frac{L}{2}} \frac{2 D_{\lambda}(t)}{H_{\lambda}(t)} d t .
$$

By Lemma 2.2,

$$
\frac{L}{2} \lambda N_{\lambda}(L) \leq-(L+\lambda) \int_{0}^{\frac{L}{2}} \frac{H_{\lambda}^{\prime}(t)}{H_{\lambda}(t)} d t=(L+\lambda) \ln \frac{H_{\lambda}(0)}{H_{\lambda}\left(\frac{L}{2}\right)} .
$$

Since

$$
\frac{H_{\lambda}(0)}{H_{\lambda}\left(\frac{L}{2}\right)} \leq \frac{\int_{\Omega}|\varphi(x, 0)|^{2} d x}{\int_{\Omega}\left|\varphi\left(x, \frac{L}{2}\right)\right|^{2} d x} \frac{\left(\frac{L}{2}+\lambda\right)^{d / 2}}{(L+\lambda)^{d / 2}} e^{\frac{m}{4\left(\frac{L}{2}+\lambda\right)}} \leq e^{\frac{m}{2 L}} \frac{\int_{\Omega}|\varphi(x, 0)|^{2} d x}{\int_{\Omega}\left|\varphi\left(x, \frac{L}{2}\right)\right|^{2} d x} .
$$

Therefore,

$$
\frac{L}{2} \lambda N_{\lambda}(L) \leq(L+\lambda)\left[\frac{m}{2 L}+\ln \frac{\int_{\Omega}|\varphi(x, 0)|^{2} d x}{\int_{\Omega}\left|\varphi\left(x, \frac{L}{2}\right)\right|^{2} d x}\right] .
$$

By direct computation, we obtain

$$
\frac{d}{d t}\left(\frac{1}{2}\|\varphi\|_{L^{2}(\Omega)}^{2}\right)=-\|\varphi\|_{H_{0}^{1}(\Omega)}^{2} \leq 0 .
$$

Thus, the solution of (1.1) satisfies that

$$
\int_{\Omega}|\varphi(x, L)|^{2} d x \leq \int_{\Omega}\left|\varphi\left(x, \frac{L}{2}\right)\right|^{2} d x
$$

We obtain (2.27). This completes the proof of this lemma.

Since $0 \in \omega$, we can get a positive number $r$ such that $B_{r} \equiv\left\{x \in \mathbb{R}^{d}:\|x\|_{\mathbb{R}^{d}} \leq r\right\} \subset \omega$. The following lemma plays a key role in the proof of the main results.

Lemma 2.6 There exists a positive number $C>1$ such that, for any $\lambda>0$,

$$
\begin{gathered}
{\left[1-\frac{8 C \lambda}{r^{2}}\left(\frac{\lambda}{L}+1\right) \mathcal{K}(L)\right] \int_{\Omega}|x|^{2}|\varphi(x, L)|^{2} e^{-\frac{|x|^{2}}{4 \lambda}} d x} \\
\leq 8 C \lambda\left(\frac{\lambda}{L}+1\right) \mathcal{K}(L) \int_{B_{r}}|\varphi(x, L)|^{2} e^{-\frac{|x|^{2}}{4 \lambda}} d x,
\end{gathered}
$$

where

$$
\mathcal{K}(L) \equiv \frac{m}{L}+2 \ln \frac{\int_{\Omega}|\varphi(x, 0)|^{2} d x}{\int_{\Omega}|\varphi(x, L)|^{2} d x}+\frac{d}{2} .
$$

Proof For any $f(x) \in H_{0}^{1}(\Omega)$, it holds that

$$
0 \leq \int_{\Omega}\left|\nabla\left(f(x) \exp \left(-\frac{|x|^{2}}{8 \lambda}\right)\right)\right|^{2} d x
$$


By direct computation, we get

$$
\int_{\Omega} \frac{|x|^{2}}{8 \lambda}|f(x)|^{2} e^{-\frac{|x|^{2}}{4 \lambda}} d x \leq 2 \lambda \int_{\Omega}|\nabla f(x)|^{2} e^{-\frac{|x|^{2}}{4 \lambda}} d x+\frac{d}{2} \int_{\Omega}|f(x)|^{2} e^{-\frac{|x|^{2}}{4 \lambda}} d x
$$

Recall that, for any $g \in H_{0}^{1}(\Omega)$, the norm $\|g\|_{1}=\left(\int_{\Omega}\left(|\nabla g|^{2}-V(x) g^{2}\right) d x\right)^{\frac{1}{2}}$ is equivalent to the standard norm in $H_{0}^{1}(\Omega)$. Thus, there exists a positive number $C>1$ such that

$$
\int_{\Omega}|\nabla g|^{2} d x \leq C \int_{\Omega}\left(|\nabla g|^{2}-V(x) g^{2}\right) d x \quad \text { for any } g \in H_{0}^{1}(\Omega) .
$$

This, combined with (2.34), shows

$$
\begin{aligned}
& \int_{\Omega}|x|^{2}|\varphi(x, L)|^{2} e^{-\frac{|x|^{2}}{4 \lambda}} d x \\
& \quad \leq 8 \lambda\left(2 \lambda C \int_{\Omega}\left[|\nabla \varphi(x, L)|^{2}-\frac{\mu|\varphi(x, L)|^{2}}{|x|^{2}}\right] e^{-\frac{|x|^{2}}{4 \lambda}} d x+\frac{d}{2} \int_{\Omega}|\varphi(x, L)|^{2} e^{-\frac{|x|^{2}}{4 \lambda}} d x\right) \\
& \quad \leq 8 \lambda\left(\lambda C N_{\lambda}(L)+\frac{d}{2}\right) \int_{\Omega}|\varphi(x, L)|^{2} e^{-\frac{|x|^{2}}{4 \lambda}} d x \\
& \quad \leq 8 \lambda\left(\lambda C N_{\lambda}(L)+\frac{d}{2}\right)\left(\int_{B_{r}}|\varphi(x, L)|^{2} e^{-\frac{|x|^{2}}{4 \lambda}} d x+\frac{1}{r^{2}} \int_{\Omega \backslash B_{r}}|x|^{2}|\varphi(x, L)|^{2} e^{-\frac{|x|^{2}}{4 \lambda}}\right) \\
& \quad \leq 8 C \lambda\left(\frac{\lambda}{L}+1\right) \mathcal{K}(L)\left(\int_{B_{r}}|\varphi(x, L)|^{2} e^{-\frac{|x|^{2}}{4 \lambda}} d x+\frac{1}{r^{2}} \int_{\Omega \backslash B_{r}}|x|^{2}|\varphi(x, L)|^{2} e^{-\frac{|x|^{2}}{4 \lambda}}\right) .
\end{aligned}
$$

Therefore,

$$
\begin{gathered}
\left(1-\frac{8 C \lambda}{r^{2}}\left(\frac{\lambda}{L}+1\right) \mathcal{K}(L)\right) \int_{\Omega}|x|^{2}|\varphi(x, L)|^{2} e^{-\frac{|x|^{2}}{4 \lambda}} \\
\leq 8 C\left(\frac{\lambda}{L}+1\right) \lambda \mathcal{K}(L) \int_{B_{r}}|\varphi(x, L)|^{2} e^{-\frac{|x|^{2}}{4 \lambda}} d x .
\end{gathered}
$$

This completes the proof of this lemma.

\section{Proof of the main result}

Proof We first prove (1.3). By taking $\lambda>0$ in estimate (2.31) to be such that

$$
\frac{8 C \lambda}{r^{2}}\left(\frac{\lambda}{L}+1\right) \mathcal{K}(L)=\frac{1}{2}
$$

By direct computation, we have

$$
\lambda=\frac{1}{2}\left(-L+\sqrt{L^{2}+\frac{L r^{2}}{4 C \mathcal{K}(L)}}\right) .
$$


Zheng et al. Journal of Inequalities and Applications

(2018) 2018:310

Page 10 of 13

Since $\frac{m}{L} \leq \mathcal{K}(L)$, it follows that

$$
\begin{aligned}
\frac{1}{\lambda} & =2 \frac{L+\sqrt{L^{2}+\frac{L r^{2}}{4 C \mathcal{K}(L)}}}{\frac{L r^{2}}{4 C \mathcal{K}(L)}} \\
& =8 C\left(L+\sqrt{L^{2}+\frac{L r^{2}}{4 C \mathcal{K}(L)}}\right) \frac{1}{L r^{2}} \mathcal{K}(L) \\
& \leq 8 C\left(2 L+\sqrt{\frac{L r^{2}}{4 C \mathcal{K}(L)}}\right) \frac{1}{L r^{2}} \mathcal{K}(L) \\
& \leq\left(16+\frac{4 r}{\sqrt{C m}}\right) \frac{C}{r^{2}} \mathcal{K}(L) .
\end{aligned}
$$

Therefore, it holds that

$$
\begin{aligned}
e^{\frac{m}{4 \lambda}} & \leq e^{\left(4 m+r \sqrt{\frac{m}{C}}\right) \frac{C}{r^{2}} \mathcal{K}(L)} \\
& \leq e^{\left(4 m+r \sqrt{\frac{m}{C}}\right) \frac{1}{r^{2}} \frac{d}{2}} e^{\left(4 m+r \sqrt{\frac{m}{C}}\right) \frac{1}{r^{2}} \frac{m}{L}}\left(\frac{\int_{\Omega}\left(|\varphi(x, 0)|^{2}\right) d x}{\int_{\Omega}\left(|\varphi(x, L)|^{2}\right) d x}\right)^{2 C\left(4 m+r \sqrt{\frac{m}{C}}\right) / r^{2}} .
\end{aligned}
$$

By Lemma 2.6, we get

$$
\int_{\Omega}|x|^{2}|\varphi(x, L)|^{2} e^{-\frac{|x|^{2}}{4 \lambda}} d x \leq r^{2} \int_{B_{r}}|\varphi(x, L)|^{2} e^{-\frac{|x|^{2}}{4 \lambda}} d x .
$$

It indicates that

$$
\begin{aligned}
\int_{\Omega}|\varphi(x, L)|^{2} e^{-\frac{m}{4 \lambda}} d x & \leq \int_{\Omega}|\varphi(x, L)|^{2} e^{-\frac{|x|^{2}}{4 \lambda}} d x \\
& =\int_{\Omega \backslash B_{r}}|\varphi(x, L)|^{2} e^{-\frac{|x|^{2}}{4 \lambda}} d x+\int_{B_{r}}|\varphi(x, L)|^{2} e^{-\frac{|x|^{2}}{4 \lambda}} d x \\
& \leq \frac{1}{r^{2}} \int_{\Omega}|x|^{2}|\varphi(x, L)|^{2} e^{-\frac{|x|^{2}}{4 \lambda}} d x+\int_{B_{r}}|\varphi(x, L)|^{2} e^{-\frac{|x|^{2}}{4 \lambda}} d x \\
& \leq 2 \int_{B_{r}}|\varphi(x, L)|^{2} e^{-\frac{|x|^{2}}{4 \lambda}} d x \leq 2 \int_{B_{r}}|\varphi(x, L)|^{2} d x .
\end{aligned}
$$

Thus,

$$
\begin{aligned}
\int_{\Omega}|\varphi(x, L)|^{2} d x \leq & 2 e^{\frac{m}{4 \lambda}} \int_{B_{r}}|\varphi(x, L)|^{2} d x \\
\leq & 2 e^{\left(4 m+r \sqrt{\frac{m}{C}}\right) \frac{1}{r^{2}} \frac{d}{2}} e^{\left(4 m+r \sqrt{\frac{m}{C}}\right) \frac{1}{r^{2}} \frac{m}{L}}\left(\frac{\int_{\Omega}\left(|\varphi(x, 0)|^{2}\right) d x}{\int_{\Omega}\left(|\varphi(x, L)|^{2}\right) d x}\right)^{2 C\left(4 m+r \sqrt{\frac{m}{C}}\right) / r^{2}} \\
& \times \int_{B_{r}}|\varphi(x, L)|^{2} d x .
\end{aligned}
$$

This shows that

$$
\int_{\Omega}|\varphi(x, L)|^{2} d x \leq C e^{\frac{C}{r^{2}}} e^{\frac{C}{L r^{2}}}\left(\frac{\int_{\Omega}|\varphi(x, 0)|^{2} d x}{\int_{\Omega}|\varphi(x, L)|^{2} d x}\right)^{C / r^{2}} \int_{B_{r}}|\varphi(x, L)|^{2} d x,
$$


which is equivalent to the following inequality:

$$
\begin{aligned}
\int_{\Omega}|\varphi(x, L)|^{2} d x & \leq C e^{\frac{C}{L}}\left(\int_{\Omega}|\varphi(x, 0)|^{2} d x\right)^{\frac{C}{r^{2}+C}}\left(\int_{B_{r}}|\varphi(x, L)|^{2} d x\right)^{\frac{r^{2}}{r^{2}+C}} \\
& \leq C e^{\frac{C}{L}}\left(\int_{\Omega}\left|\varphi_{0}(x)\right|^{2} d x\right)^{\frac{C}{r^{2}+C}}\left(\int_{\omega}|\varphi(x, L)|^{2} d x\right)^{\frac{r^{2}}{r^{2}+C}} .
\end{aligned}
$$

Let $\alpha=\frac{r^{2}}{r^{2}+C}$, then the above inequality can be written as

$$
\int_{\Omega}|\varphi(x, L)|^{2} d x \leq C e^{\frac{C}{L}}\left(\int_{\Omega}\left|\varphi_{0}(x)\right|^{2} d x\right)^{1-\alpha}\left(\int_{\omega}|\varphi(x, L)|^{2} d x\right)^{\alpha} .
$$

Conclusion (1.3) then follows.

In order to prove (1.4), we will prove the following estimate:

$$
\|\varphi(x, 0)\|_{H^{-1}(\Omega)}^{2} \leq \exp \left(C L \frac{\|\varphi(x, 0)\|_{H^{-1}(\Omega)}^{2}}{\|\varphi(x, 0)\|_{H^{-1}(\Omega)}^{2}}\right)\|\varphi(x, L)\|_{H^{-1}(\Omega)}^{2} .
$$

We define a function $\Phi(t)$ as follows:

$$
\Phi(t)=\frac{\|\varphi(x, t)\|_{L^{2}(\Omega)}^{2}}{\|\varphi(x, t)\|_{H^{-1}(\Omega)}^{2}} .
$$

By direct computation, we obtain

$$
\frac{d}{d t}\left(\frac{1}{2}\|\varphi\|_{H^{-1}(\Omega)}^{2}\right)=-\|\varphi\|_{L^{2}(\Omega)}^{2} .
$$

This, together with (2.4) and (2.29), indicates

$$
\begin{aligned}
\frac{d}{d t} \Phi(t) & =\frac{\left(\|\varphi\|_{L^{2}(\Omega)}^{2}\right)^{\prime}\left(\|\varphi\|_{H^{-1}(\Omega)}^{2}\right)-\left(\|\varphi\|_{L^{2}(\Omega)}^{2}\right)\left(\|\varphi\|_{H^{-1}(\Omega)}^{2}\right)^{\prime}}{\left(\|\varphi\|_{H^{-1}(\Omega)}^{2}\right)^{2}} \\
& =\frac{2}{\left(\|\varphi\|_{H^{-1}(\Omega)}^{2}\right)^{2}}\left\{-\|\varphi\|_{H_{0}^{1}(\Omega)}^{2}\|\varphi\|_{H^{-1}(\Omega)}^{2}+\|\varphi\|_{L^{2}(\Omega)}^{4}\right\} \leq 0 .
\end{aligned}
$$

Thus, $\Phi(t)$ is a decreasing function, and

$$
\Phi(L) \leq \Phi(0) .
$$

It follows from (2.29) and (3.6) that

$$
\begin{aligned}
0 & =\frac{1}{2} \frac{d}{d t}\left(\|\varphi\|_{H^{-1}(\Omega)}^{2}\right)+\|\varphi\|_{L^{2}(\Omega)}^{2} \\
& \leq \frac{1}{2} \frac{d}{d t}\left(\|\varphi\|_{H^{-1}(\Omega)}^{2}\right)+\Phi(0)\|\varphi\|_{H^{-1}(\Omega)}^{2} .
\end{aligned}
$$

Integrating (3.7) on $(0, L)$, we get the desired estimate

$$
\|\varphi(x, 0)\|_{H^{-1}(\Omega)}^{2} \leq e^{2 \Phi(0) L}\|\varphi(x, L)\|_{H^{-1}(\Omega)}^{2} .
$$

With the aid of (3.5), we can get (1.4). This completes the proof. 
Corollary 3.1 Suppose that $\omega$ is a non-empty open subset of $\Omega, 0 \in \omega$, and $\varphi_{0} \in L^{2}(\Omega)$. Then there exist two positive numbers $\alpha=\alpha(\Omega, \omega), C=C(\Omega, \omega)$ such that, for each $L>0$ and $\tilde{\Omega} \Subset \Omega$,

$$
\int_{\tilde{\Omega}}|\varphi(x, L)|^{2} d x \leq C e^{\frac{2 C}{L}} L^{\alpha-1}\left(\|\varphi(x, s)\|_{L^{2}(\Omega \times(0, L))}\right)^{1-\alpha}\left(\int_{\omega}|\varphi(x, L)|^{2} d x\right)^{\alpha} .
$$

Proof For any $s \in\left[0, \frac{L}{2}\right]$, we take $z(x, t)=\varphi(x, t+s)$, where $t \in[0, L-s], x \in \Omega$. Then $z(t, x)$ satisfies the following equation:

$$
\begin{cases}\partial_{t} z(x, t)-\Delta z(x, t)-V(x) z(x, t)=0 & \text { in } \Omega \times(0, L-s] \\ z(x, t)=0 & \text { on } \partial \Omega \times(0, L-s], \\ z(x, 0)=\varphi(s, x) & \text { in } \Omega .\end{cases}
$$

By the same argument as that in the proof of Theorem 1.1, we also get

$$
\int_{\Omega}|z(x, L-s)|^{2} d x \leq C e^{\frac{C}{L-s}}\left(\int_{\Omega}|z(x, 0)|^{2} d x\right)^{1-\alpha}\left(\int_{\omega}|z(x, L-s)|^{2} d x\right)^{\alpha}
$$

where the constant $C$ is a positive constant only depending on $\Omega$ and $\omega$.

Thus,

$$
\int_{\Omega}|\varphi(x, L)|^{2} d x \leq C e^{\frac{C}{L-s}}\left(\int_{\Omega}|\varphi(x, s)|^{2} d x\right)^{1-\alpha}\left(\int_{\omega}|\varphi(x, L)|^{2} d x\right)^{\alpha} .
$$

Then we have

$$
\begin{aligned}
\frac{L}{2} \int_{\Omega}|\varphi(x, L)|^{2} d x & =\int_{0}^{\frac{L}{2}} \int_{\Omega}|\varphi(x, L)|^{2} d x d s \\
& \leq \int_{0}^{\frac{L}{2}} \operatorname{Ce}^{\frac{C}{L-s}}\left(\int_{\Omega}|\varphi(x, s)|^{2} d x\right)^{1-\alpha}\left(\int_{\omega}|\varphi(x, L)|^{2} d x\right)^{\alpha} d s \\
& \leq C e^{\frac{2 C}{L}}\left(\int_{\omega}|\varphi(x, L)|^{2} d x\right)^{\alpha} \int_{0}^{\frac{L}{2}}\left(\int_{\Omega}|\varphi(x, s)|^{2} d x\right)^{1-\alpha} d s \\
& \leq C e^{\frac{2 C}{L}}\left(\frac{L}{2}\right)^{\alpha}\left(\int_{\omega}|\varphi(x, L)|^{2} d x\right)^{\alpha}\left(\|\varphi(x, s)\|_{L^{2}\left(\Omega \times\left(0, \frac{L}{2}\right)\right)}\right)^{1-\alpha} .
\end{aligned}
$$

The last step is obtained by Hölder's inequality. Therefore, we can get (3.8). This completes the proof.

\section{Acknowledgements}

The authors would like to express their sincere thanks to the referees for their valuable suggestions.

\section{Funding}

This work was partially supported by the National Natural Science Foundation of China (11501178), the Natural Science Foundation of Henan Province (No. 162300410176). 
Authors' contributions

$\mathrm{GZ}$ provided the question. $\mathrm{GZ}, \mathrm{KL}$, and $\mathrm{YZ}$ gave the proof for the main results together. All authors read and approved the final manuscript.

\section{Author details}

${ }^{1}$ College of Mathematics and Information Science, Henan Normal University, Xinxiang, P.R. China. ${ }^{2}$ Xinxiang No. 7 Middle School, Xinxiang, P.R. China.

\section{Publisher's Note}

Springer Nature remains neutral with regard to jurisdictional claims in published maps and institutional affiliations.

Received: 30 July 2018 Accepted: 8 November 2018 Published online: 14 November 2018

\section{References}

1. Almgren, F.J. Jr.: Dirichlet's problem for multiple valued functions and the regularity of mass minimizing integral currents. In: Minimal Submanifolds and Geodesics, pp. 1-6. North-Holland, Amsterdam (1979)

2. Azorero, J.P.G., Alonso, I.P.: Hardy inequalities and some critical elliptic and parabolic problems. J. Differ. Equ. 144 441-476 (1998)

3. Baras, P., Goldstein, J.A.: The heat equation with a singular potential. Trans. Am. Math. Soc. 284, 121-139 (1984)

4. Brezis, H., Marcus, M.: Hardy's inequality revisited. Ann. Sc. Norm. Super. Pisa, Cl. Sci. 25, 217-237 (1997)

5. Escauriaza, L., Fernandez, F.J., Vessella, S.: Doubling properties of caloric functions. Appl. Anal. 85, 205-223 (2006)

6. Garofalo, N., Lin, F.H.: Monotonicity properties of variational integrals, $A_{p}$ weights and unique continuation. Indiana Univ. Math. J. 35, 245-267 (1986)

7. Goldstein, J.A., Zhang, Q.S.: Linear parabolic equations with strong singular potentials. Trans. Am. Math. Soc. 355, 197-211 (2003)

8. Kenig, C.: Quantitative unique continuation, logarithmic convexity of Gaussian means and Hardy's uncertainty principle. Proc. Symp. Pure Math. 79, 207-227 (2008)

9. Koch, H., Tataru, D.: Carleman estimates and unique continuation for second order parabolic equations with nonsmooth coefficients. Commun. Partial Differ. Equ. 34(4), 305-366 (2009)

10. Landis, E.M., Oleinik, O.A.: Generalized analyticity and some related properties of solutions of elliptic and parabolic equations. Russ. Math. Surv. 29, 195-212 (1974)

11. Lin, F.: A uniqueness theorem for parabolic equations. Commun. Pure Appl. Math. 43, 127-136 (1990)

12. Moroz, S., Schmidt, R.: Nonrelativistic inverse square potential, scale anomaly, and complex extension. Ann. Phys. 325 491-513 (2010)

13. Peral, I., Vazquez, J.L.: On the stability or instability of the singular solution of the semilinear heat equation with exponential reaction term. Arch. Ration. Mech. Anal. 129, 201-224 (1995)

14. Phung, K.D., Wang, G.: Quantitative unique continuation for the semilinear heat equation in a convex domain J. Funct. Anal. 259(5), 1230-1247 (2010)

15. Phung, K.D., Wang, L.J., Zhang, C.: Bang-bang property for time optimal control of semilinear heat equation. Ann. Inst Henri Poincaré, Anal. Non Linéaire 31, 477-499 (2014)

16. Poon, C.: Unique continuation for parabolic equations. Commun. Partial Differ. Equ. 21, 521-539 (1996)

17. Reed, M., Simon, B.: Methods of Modern Mathematical Physics III. Elsevier, Singapore (2003)

18. Tucsnak, M., Weiss, G.: Observation and Control for Operator Semigroups. Birkhäuser, Basel (2009)

19. Vazquez, J.L., Zuazua, E.: The Hardy inequality and the asymptotic behaviour of the heat equation with an inverse-square potential. J. Funct. Anal. 173, 103-153 (2000)

\section{Submit your manuscript to a SpringerOpen ${ }^{\circ}$ journal and benefit from:}

- Convenient online submission

- Rigorous peer review

- Open access: articles freely available online

- High visibility within the field

- Retaining the copyright to your article

Submit your next manuscript at $\boldsymbol{s p r i n g e r o p e n . c o m ~}$ 